OPEN ACCESS

Edited by:

Maurizio Muscaritoli,

Sapienza Università di Roma, Italy

Reviewed by:

Antonietta Gigante,

Sapienza University of Rome, Italy

Fernando Rodriguez-Artalejo,

Autonomous University of

Madrid, Spain

*Correspondence: Dan-qing Yu

gdydq100@126.com

Specialty section: This article was submitted to

Clinical Nutrition,

a section of the journal

Frontiers in Nutrition

Received: 25 November 2021

Accepted: 31 January 2022

Published: 23 February 2022

Citation:

Yang Z-w, Wei X-b, Fu B-q, Chen J-y and Yu D-q (2022) Prevalence and Prognostic Significance of Malnutrition in Hypertensive Patients in a Community Setting

Front. Nutr. 9:822376. doi: 10.3389/fnut.2022.822376

\section{Prevalence and Prognostic Significance of Malnutrition in Hypertensive Patients in a Community Setting}

\author{
Zhi-wen Yang ${ }^{1,2}$, Xue-biao Wei ${ }^{2,3}$, Bing-qi Fu ${ }^{1,2}$, Ji-yan $\mathrm{Chen}^{2}$ and Dan-qing $\mathrm{Yu}^{2 *}$ \\ ${ }^{1}$ Shantou University Medical College, Shantou, China, ${ }^{2}$ Division of Cardiology, Guangdong Cardiovascular Institute, \\ Guangdong Provincial Key Laboratory of Coronary Heart Disease Prevention, Guangdong Provincial People's Hospital, \\ Guangdong Academy of Medical Sciences, Guangzhou, China, ${ }^{3}$ Division of Geriatrics Intensive Medicine, Guangdong \\ Provincial Geriatrics Institute, Guangdong Provincial People's Hospital, Guangdong Academy of Medical Sciences, \\ Guangzhou, China
}

Background: Malnutrition is a significantly poor prognostic factor for a variety of cardiovascular diseases. However, its prevalence and prognostic value in hypertensive patients is still unclear. The present study sought to determine the prevalence and prognostic value of malnutrition in hypertensive patients in a community setting.

Methods: We included 9,949 hypertensive patients from the National Health and Nutrition Examination Survey (NHANES) (2005-2014). The Controlling Nutritional Status (CONUT) score, the Nutritional Risk Index (NRI), and the Naples Prognostic Score (NPS) were applied to assess the nutritional status of participants. A Cox regression model was established to examine the association between malnutrition and cardiovascular and all-cause mortality.

Results: In all, 19.9, 3.9, and 82.9\% hypertensive patients were considered to have malnutrition as evaluated by the CONUT, NRI, and NPS, respectively. Malnutrition assessed by CONUT and NRI was independently associated with cardiovascular mortality ( $\mathrm{HR}[95 \% \mathrm{Cl}]$ ) for mild and moderate-to-severe degree of malnutrition, respectively: 1.41 (1.04-1.91) and 5.79 (2.34-14.29) for CONUT; 2.60 (1.34-5.07) and 3.30 (1.66-6.56) for $\mathrm{NRI}$ (all $P<0.05)$, and for all-cause mortality $(\mathrm{HR}[95 \% \mathrm{Cl}]$ ) for mild and moderate-to-severe degree of malnutrition, respectively: 1.48 (1.30-1.70) and 4.87 (3.40-6.98) for CONUT; 1.72 (1.24-2.39) and 2.60 (1.96-3.44) for NRI (all $P<0.01$ ). Naples Prognostic Score could only independently predict all-cause mortality.

Conclusions: Malnutrition was common among hypertensive patients and was closely associated with both long-term cardiovascular and all-cause mortality.

Keywords: malnutrition, hypertension, Controlling Nutritional Status (CONUT) score, Nutritional Risk Index (NRI), Naples Prognostic Score, cardiovascular mortality, all-cause mortality 


\section{INTRODUCTION}

The effect of nutritional state on a variety of cardiovascular diseases is now the subject of increasing concern, as it is modifiable compared to other clinical variables (1). Most previous studies were focused on overnutrition and the results suggested that it was a significant risk factor for cardiovascular disease (2). However, recent studies have reported that malnutrition is a significantly poor prognostic factor of acute coronary artery disease, heart failure, atrial fibrillation, and valvular heart disease (3-5).

Hypertension, one of the most commonly occurring diseases worldwide (6), contributes to the risk of developing coronary heart disease, stroke, and other cardiovascular disease (7). The aged-standardized prevalence of hypertension reported on 2015 was $24.1 \%$ for men and $20.1 \%$ for women globally (8). Moreover, nutritional factors such as nutrient intake, blood lipids, and high Body Mass Index (BMI) have been shown to be associated with blood pressure control and mortality $(9,10)$. However, less attention has been paid to the prevalence and prognostic value of malnutrition among hypertensive patients.

Thus, we aimed to determine the prevalence and prognostic value of malnutrition among hypertensive patients in a community setting by using three nutritional screening tools (NSTs), namely Controlling Nutritional Status (CONUT), Nutritional Risk Index (NRI), and Naples Prognostic Score (NPS).

\section{METHODS}

\section{Study Population}

This retrospective observational study was based on National Health and Nutrition Examination Survey (NHANES) (20052014) (11) - a large nationwide survey on the civilian US population conducted by the National Center for Health Statistics of the Centers for Disease Control and Prevention. All NHANES study protocols survey protocol was approved by the Ethics Review Committee of NCHS of the Centers for Disease Control and Prevention. All participants had provided written, informed consent for the use of their data. All procedures in this study were conducted in accordance with all the relevant guidelines. We included participants aged $\geq 18$ years with hypertension. However, individuals with missing data on lymphocyte count $(n=888)$, serum albumin $(n=1,060)$, serum total cholesterol $(n=1,017)$, and height and weight $(n=532)$ was excluded, leaving 9,949 participants for the final analysis (Figure 1).

\section{Baseline Assessment}

The data on physical examination, questionnaires, and laboratory examination was obtained from NHANES, which were performed in a standardized manner. Covariates including sociodemographic information; current smoking status; current alcohol drinking, medical history (congestive heart disease, coronary heart disease, diabetes, stroke, emphysema, liver disease, and malignant tumor); BMI; hemoglobin; albuminuria; and estimated glomerular filtration rate (eGFR) were assessed. Body Mass Index was defined as the weight in kilograms divided by the square of height in meters. Albuminuria was defined by urinary albumin creatine ratio $\geq 30 \mathrm{mg} / \mathrm{g}$ and eGFR was calculated using the Chronic Kidney Disease Epidemiology Collaboration equation.

\section{Definition of Hypertension}

Hypertension and antihypertensive medication history were collected by questionnaires. Blood pressure was obtained with a mercury sphygmomanometer with an appropriately sized cuff by a trained physician. Blood pressure measurement was performed three times and the average value of the three measurements was defined as the systolic blood pressure (SBP) and diastolic blood pressure (DBP). Hypertension was defined as having a self-reported hypertension history or using antihypertensive medications or SBP $\geq 140 \mathrm{mmHg}$ or DPB $\geq 90 \mathrm{mmHg}$.

\section{Nutrition Status Assessment}

The CONUT score (12), calculated based on the levels of serum albumin, total cholesterol, and lymphocytes, was developed as a screening tool for early detection of malnutrition. We categorized the scores into three groups: normal, $0-1$; mild, 2-4; and moderate-to-severe, 5-12.

The NRI (13), a popular nutrition screening tool in recent years, was originally defined as $1.519 \times$ serum albumin $(\mathrm{g} / \mathrm{l})+$ $41.7 \times$ (current body weight $[\mathrm{kg}] /$ usual body weight $[\mathrm{kg}])$. The actual body weight was usually replaced by ideal body weight which is defined as height $(\mathrm{cm})-100-([$ height $(\mathrm{cm})-150] / 2.5)$ for women and height $(\mathrm{cm})-100-([$ height $(\mathrm{cm})-150] / 4)$ for men. Patients were categorized into three groups according to their NRI: no nutritional risk (NRI $\geq 100$ ), mild nutritional risk $(97.5 \leq \mathrm{NRI}<100)$, and moderate-to-severe nutritional risk $(\mathrm{NRI}<97.5)$.

The NPS (14), a tool to access the nutritional and inflammatory status of patients, is often used among patients with malignancies. The NPS takes into account serum albumin $(\mathrm{mg} / \mathrm{dl})$, total cholesterol $(\mathrm{mg} / \mathrm{dl})$, the neutrophil:lymphocyte ratio, and lymphocyte:monocyte ratio. A score of 0 is considered normal; scores of 1-2 and 3-4 reflect mild and moderate-tosevere malnutrition, respectively.

\section{Outcomes}

The endpoints were long-term cardiovascular or all-cause mortality. The mortality status of participants was obtained by data matching with death certificates in the National Death Index until December 31, 2015. Cardiovascular death was determined based on the International Classification of Diseases, 10th Edition, Clinical Modification System codes (I00-I09, I11, I13, I20-I51).

\section{Statistical Analysis}

Baseline characteristics were expressed as a median with interquartile range $\left(25^{\text {th }}-75^{\text {th }}\right.$ percentiles $)$ for continuous variables and with categorical data expressed as $n$ (\%). Venn diagrams were used to illustrate the relationship 


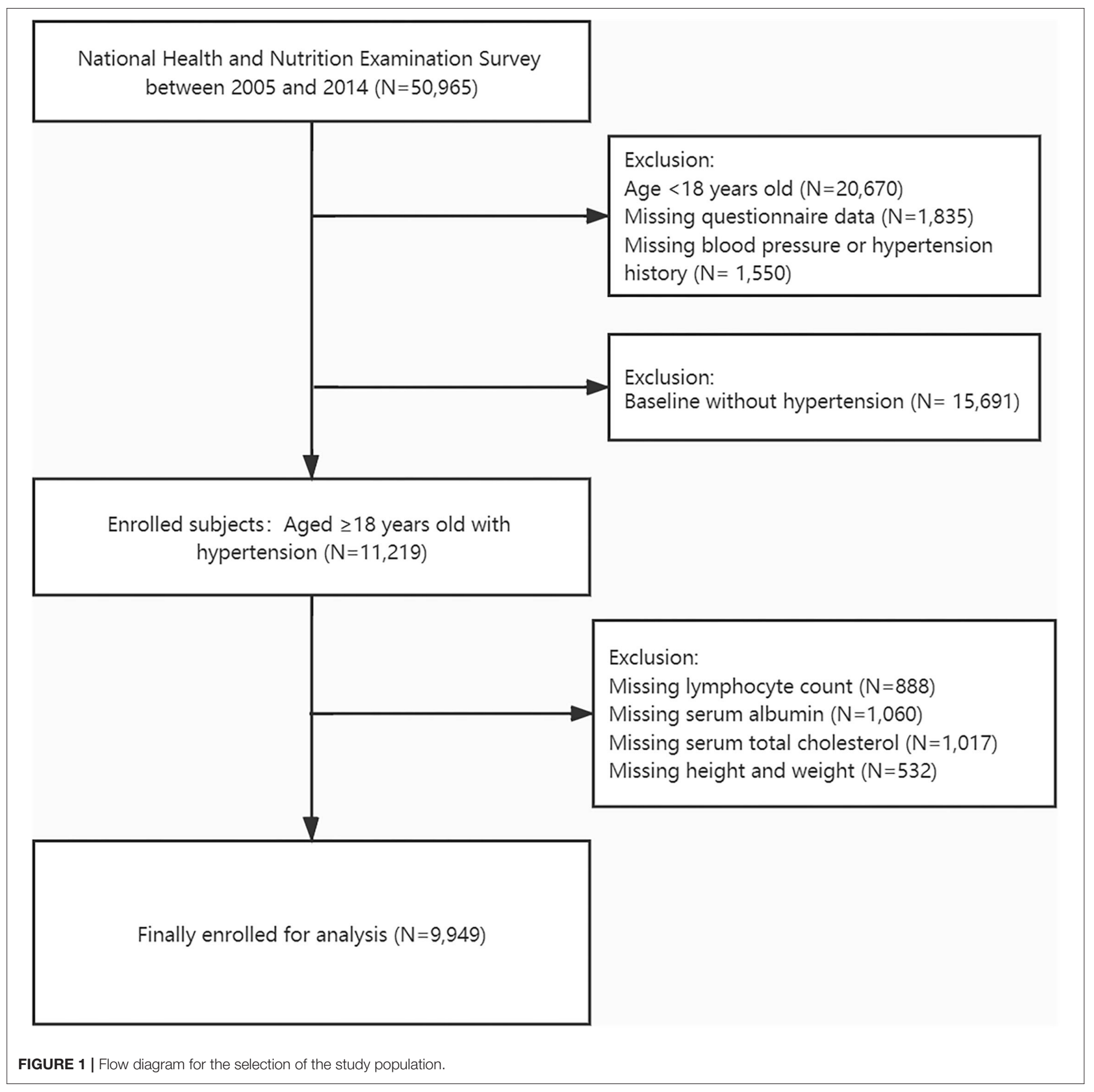

between the three malnutritional indices. Survival analysis was performed with standardized Kaplan-Meier curves and the log-rank test. Cox proportional hazards regression models were used to estimate hazard ratios (HRs) and 95\% confidence intervals (CIs) for cardiovascular and all-cause mortality.

All statistical analyses were performed using SPSS v25.0 (IBM Corporation, Armonk, NY, USA) and eulerAPE v3 (15). A two-sided $P<0.05$ was considered to indicate statistical significance.

\section{RESULTS}

\section{Baseline Characteristics of the Study Population}

The baseline characteristics of participants are summarized in Table 1. The analysis included 9,949 hypertensive patients with a mean age of $49.1 \pm 17.8$ years; $50.1 \%$ subjects were female. Overall, $12.8 \%$ participants died during the survey with a mean follow-up time of 5.48 years; of these, 244 (2.5\%) participants died from cardiovascular causes. 
TABLE 1 | Baseline clinical characteristics of included patients.

\begin{tabular}{|c|c|}
\hline Variables & Total $(n=9,949)$ \\
\hline Age, years & $59.64 \pm 15.20$ \\
\hline Female gender, $n(\%)$ & 4,992 (50.2) \\
\hline \multicolumn{2}{|l|}{ Ethnicity, $n$ (\%) } \\
\hline Non-white & $4,750(47.7)$ \\
\hline White & $5,199(52.3)$ \\
\hline $\mathrm{BMl}$ & $30.6 \pm 7.2$ \\
\hline \multicolumn{2}{|l|}{ BMI classification } \\
\hline Underweight & $97(1.0)$ \\
\hline Normal & 1,908 (19.2) \\
\hline Overweight & $3,251(32.7)$ \\
\hline Obesity & 4,693 (47.2) \\
\hline Current smoke, $n(\%)$ & 1,896 (19.1) \\
\hline Diabetes mellitus, $n(\%)$ & $2,136(22.1)$ \\
\hline Stroke, $n(\%)$ & $710(7.2)$ \\
\hline Emphysema, $n(\%)$ & $321(3.2)$ \\
\hline Liver disease, $n$ (\%) & $486(4.9)$ \\
\hline Malignant tumor, $n$ (\%) & 1,367 (13.8) \\
\hline Congestive heart failure, $n(\%)$ & $608(6.1)$ \\
\hline Coronary heart disease, $n(\%)$ & $763(7.7)$ \\
\hline Albuminuria, $n(\%)$ & 1,949 (19.9) \\
\hline Hemoglobin (g/L) & $13.98 \pm 1.58$ \\
\hline Serum albumin (g/L) & $41.84 \pm 3.32$ \\
\hline Lymphocyte count $\left(10^{9} / \mathrm{L}\right)$ & $2.12 \pm 1.14$ \\
\hline Total cholesterol (mmol/L) & $5.06 \pm 1.12$ \\
\hline eGFR(ml/min/1.73 m²) & $79.11 \pm 23.00$ \\
\hline Follow-up time, years & $5.47 \pm 2.82$ \\
\hline \multicolumn{2}{|l|}{ Long-term mortality ${ }^{\star}, n$ (\%) } \\
\hline All-cause & $1,254(12.6)$ \\
\hline Cardiovascular & $241(2.7)$ \\
\hline
\end{tabular}

BMI, Body Mass Index; eGFR, estimated Glomerular Filtration Rate.

\section{Prevalence and Clinical Feature of Malnutrition}

The percentage of malnutrition was $19.9,3.9$, and $82.9 \%$ as evaluated by CONUT, NRI, and NPS, respectively. Moreover, the percentage of moderate-to-severe malnutrition varied from $0.7 \%$ with CONUT, $2.2 \%$ with NRI, and $15.4 \%$ with NPS (Table 2). The correlation between three NSTs was weak but significant (CONUT vs. NRI: $r=0.137, P<0.01$; CONUT vs. NPS: $r=0.226, P<0.01$; NRI vs. NPS: $r=0.063, P<$ 0.01 Figure 2). Patients with malnutrition assessed by any of the three NSTs were older, with lower BMI and hemoglobin level, and worse renal function and more comorbidities than those with normal nutritional status. The former group of patients also had higher all-cause and cardiovascular mortality (Table 3).

\section{Malnutrition Score, All-Cause Mortality and Cardiovascular Mortality}

As shown by univariate Cox proportional hazard regression (Tables 4, 5) and Kaplan-Meier survival curves (Figure 3),
TABLE 2 | Prevalence of malnutrition according to three nutritional screening tools.

\begin{tabular}{llc}
\hline & Nutritional indices & Total $(\boldsymbol{n}=\mathbf{9 , 9 4 9 )}$ \\
\hline CONUT & Normal, $n(\%)$ & $7,950(79.7)$ \\
& Mild, $n(\%)$ & $1,934(19.4)$ \\
& Moderate to severe, $n(\%)$ & $65(0.7)$ \\
$\mathrm{NRI}$ & Normal, $n(\%)$ & $9,566(96.2)$ \\
& Mild, $n(\%)$ & $164(1.6)$ \\
NPS & Moderate to severe, $n(\%)$ & $219(2.2)$ \\
& Normal, $n(\%)$ & $1,691(17.0)$ \\
& Mild, $n(\%)$ & $6,700(67.3)$ \\
& Moderate to severe, $n(\%)$ & $1,558(15.7)$ \\
\hline
\end{tabular}

CONUT, Controlling Nutritional Status score; NRI, Nutritional Risk Index; NPS, Naples Prognostic Score.

compared to normal nutritional status, worse nutritional status evaluated by any of three NSTs in both continuous form and categorical form tended to have a higher cardiovascular mortality and all-cause mortality. After adjusting for variables such as age, sex, renal insufficiency, and other diseases that could have influenced long-term mortality in univariate Cox regression analyses (Table 4), malnutrition evaluated by NPS was not associated with higher incidence of cardiovascular death (mild: adjusted HR $=1.08,95 \% \mathrm{CI}: 0.69-1.73, P=$ 0.76; moderate-to-severe: adjusted $\mathrm{HR}=1.54$, 95\% CI: $0.91-$ 2.63, $P=0.11$ Table 5; Figure 4), but it was still significant for all-cause mortality prediction (mild: adjusted $\mathrm{HR}=$ 1.65, 95\% CI: 1.30-2.08, $P<0.01$; moderate-to-severe: adjusted HR $=2.90,95 \%$ CI: 2.24-3.74, $P<0.01)$. Expect for NPS, both CONUT and NRI could independently predict cardiovascular mortality (adjusted HR (95\%CI) for mild and moderate-to-severe degree of malnutrition, respectively: $1.41(1.04-1.91)$ and $5.79(2.34-14.29)$ for CONUT; 2.60 (1.34-5.07) and $3.30(1.66-6.56)$ for NRI, all $P<0.05)$ and all-cause mortality (adjusted HR (95\%CI) for mild and moderate-to-severe degree of malnutrition, respectively: 1.48 (1.30-1.70) and $4.87(3.40-6.98)$ for CONUT; 1.72 (1.242.39) and $2.60(1.96-3.44)$ for NRI, all $P<0.01)$ and further adjustment for educational level, family income, current smoking, alcohol intake, and diet health also showed a similar result (Supplementary Tables 1A,B). By further stratified by age, chronic disease, and blood pressure control, NRI could only independently predict cardiovascular mortality and all-cause mortality in hypertensive patient with aged over 60 or comorbidities (Supplementary Tables 2A,B) and had advantages on predicting cardiovascular mortality among hypertensive patients with controlled blood pressure (Supplementary Table 2C).

\section{DISCUSSION}

In this study, we reported the prevalence and prognostic value of malnutrition among 9,949 hypertensive patients in a community setting by three NSTs. Our research showed that malnutrition as 

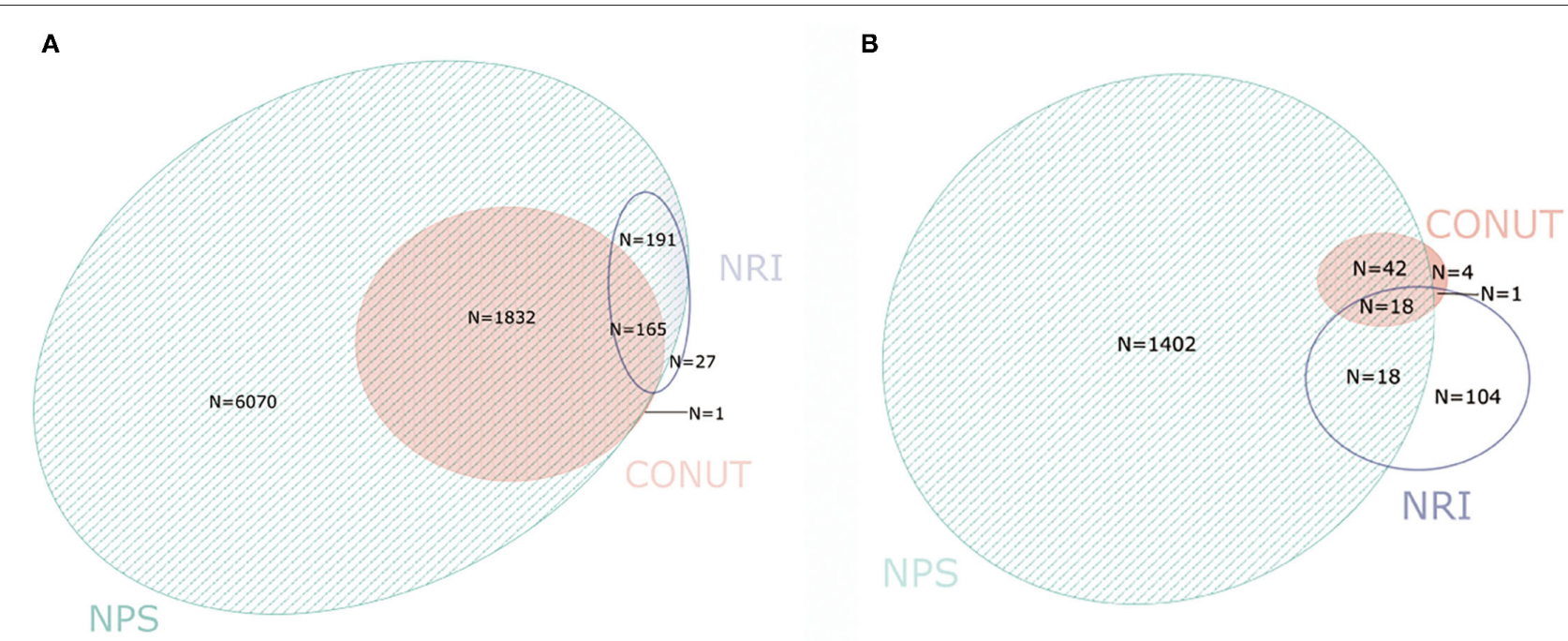

FIGURE 2 | Venn diagram. The numbers in circles indicate the cumulative frequency of malnutrition [any degree (A) vs. moderate-severe (B)] according to each malnutrition screening tool. The overlapping area of the circles indicate the patient which diagnosed with malnutrition by more than one malnutrition screening tool. CONUT, Controlling Nutritional Status score; NRI, Nutritional Risk Index; NPS, Naples Prognostic Score.

TABLE 3 | Comparison of characteristics of study population by different nutritional status.

\begin{tabular}{|c|c|c|c|c|c|c|c|c|c|}
\hline \multirow[t]{2}{*}{ Variables } & \multicolumn{3}{|c|}{ COUNT } & \multicolumn{3}{|c|}{ NPS } & \multicolumn{3}{|c|}{ NRI } \\
\hline & Normal & Malnutrition & $P$ & Normal & Malnutrition & $P$ & Normal & Malnutrition & $P$ \\
\hline Age, years & $58.5 \pm 14.9$ & $64.3 \pm 15.4$ & $<0.01$ & $55.5 \pm 13.7$ & $60.5 \pm 15.4$ & $<0.01$ & $59.3 \pm 15.1$ & $67.2 \pm 14.8$ & $<0.01$ \\
\hline Female gender, $n(\%)$ & 4,226 (53.2) & 766 (38.3) & $<0.01$ & $1,040(61.5)$ & 3,952 (47.9) & $<0.01$ & $4,785(50.0)$ & 207 (54.0) & 0.14 \\
\hline Ethnicity(white), $n$ (\%) & 3,703 (46.6) & 1,047 (52.4) & $<0.01$ & $579(34.2)$ & $4,171(50.5)$ & $<0.01$ & $4,569(47.8)$ & $181(47.3)$ & 0.88 \\
\hline BMl & $30.8 \pm 7.1$ & $30.0 \pm 7.3$ & $<0.01$ & $30.4 \pm 6.8$ & $30.7 \pm 7.2$ & 0.11 & $31.0 \pm 7.0$ & $21.0 \pm 2.8$ & $<0.01$ \\
\hline Diabetes mellitus, $n(\%)$ & $1,508(19.7)$ & 615 (31.9) & $<0.01$ & $282(17.3)$ & $1,841(23.2)$ & $<0.01$ & $2,064(22.4)$ & $59(25.6)$ & $<0.01$ \\
\hline Stroke, $n(\%)$ & $492(6.2)$ & $281(10.9)$ & $<0.01$ & $79(4.7)$ & $631(7.7)$ & $<0.01$ & $657(6.9)$ & $53(13.9)$ & $<0.01$ \\
\hline Emphysema, n (\%) & $221(2.8)$ & $100(5.0)$ & $<0.01$ & $32(1.9)$ & $289(3.5)$ & $<0.01$ & $287(3.0)$ & $34(8.9)$ & $<0.01$ \\
\hline Liver disease, $n$ (\%) & $370(4.7)$ & $116(5.8)$ & 0.04 & $66(3.9)$ & $420(5.1)$ & 0.04 & 467 (4.9) & $19(5.0)$ & 1.00 \\
\hline Malignant tumor, $n$ (\%) & $944(11.9)$ & $423(21.2)$ & $<0.01$ & $134(7.9)$ & $1,233(14.9)$ & $<0.01$ & $1,293(13.5)$ & $74(19.3)$ & $<0.01$ \\
\hline Congestive heart failure, $n(\%)$ & $370(4.7)$ & $238(12.0)$ & $<0.01$ & $48(2.8)$ & $560(6.8)$ & $<0.01$ & $576(6.0)$ & $32(8.4)$ & 0.07 \\
\hline Coronary heart disease, $n(\%)$ & $475(6.0)$ & $288(14.6)$ & $<0.01$ & $52(3.1)$ & $711(8.7)$ & $<0.01$ & $737(7.8)$ & $26(6.9)$ & 0.59 \\
\hline Albuminuria, $n(\%)$ & $1,425(18.1)$ & $524(21.7)$ & $<0.01$ & $248(14.7)$ & $1,701(21.0)$ & $<0.01$ & $1,833(19.4)$ & $116(32.0)$ & $<0.01$ \\
\hline Hemoglobin (g/L) & $14.1 \pm 1.5$ & $13.5 \pm 1.7$ & $<0.01$ & $14.1 \pm 1.4$ & $14.0 \pm 1.6$ & $<0.01$ & $14.0 \pm 1.6$ & $13.0 \pm 1.6$ & $<0.01$ \\
\hline eGFR(ml/min/1.73m²) & $80.9 \pm 22.0$ & $71.9 \pm 25.5$ & $<0.01$ & $85.3 \pm 19.9$ & $77.8 \pm 23.4$ & $<0.01$ & $79.5 \pm 22.8$ & $70.4 \pm 26.7$ & $<0.01$ \\
\hline \multicolumn{10}{|l|}{ Long-term mortality, (\%) } \\
\hline All-cause & $801(10.1)$ & $453(22.7)$ & $<0.01$ & $90(5.3)$ & $1,164(14.1)$ & $<0.01$ & $1,119(11.7)$ & $135(35.2)$ & $<0.01$ \\
\hline Cardiovascular & $159(2.2)$ & $82(5.0)$ & $<0.01$ & $24(1.5)$ & $217(3.0)$ & $<0.01$ & $216(2.5)$ & $25(9.2)$ & $<0.01$ \\
\hline
\end{tabular}

BMI, Body Mass Index; eGFR, estimated Glomerular Filtration Rate; CONUT, Controlling Nutritional Status score; NRI, Nutritional Risk Index; NPS, Naples Prognostic Score.

assessed by NSTs was common among hypertensive patients and associated with both cardiovascular and all-cause mortality.

Hypertension is considered a nutritional factor-related disease $(16,17)$. Few studies have analyzed the prevalence of malnutrition in hypertensive patients. Sun et al. (18) reported that the prevalence of malnutrition in elderly patients with hypertension was 52.4 and $27.1 \%$ for mild malnutrition and moderate-to-severe malnutrition, respectively, using the
CONUT scoring system in a cohort of 336 patients aged $\geq 80$ years. In our study of hypertensive patients, the percentage of individuals with malnutrition assessed by CONUT, NRI, and NPS was 19.9, 3.9, and 82.9\%, respectively. Moreover, $0.7,2.2$, and $15.4 \%$ of individuals with hypertension were classified as having moderate-to-severe malnutrition assessed by CONUT, NRI, and NPS, respectively. The remarkable difference in malnutrition prevalence among the three screening tools 
TABLE 4 | Univariate Cox regression for long-term mortality.

\begin{tabular}{|c|c|c|c|c|c|c|}
\hline \multirow[t]{2}{*}{ Variables } & \multicolumn{3}{|c|}{ Cardiovascular death } & \multicolumn{3}{|c|}{ All cause death } \\
\hline & HR & $95 \% \mathrm{Cl}$ & $\boldsymbol{P}$ & HR & $95 \% \mathrm{Cl}$ & $P$ \\
\hline Age & 1.10 & $1.09-1.12$ & $<0.01$ & 1.08 & $1.08-1.09$ & $<0.01$ \\
\hline Female gender & 0.59 & $0.46-0.77$ & $<0.01$ & 0.79 & $0.70-0.88$ & $<0.01$ \\
\hline White race & 1.79 & $1.38-2.32$ & $<0.01$ & 1.62 & $1.45-1.82$ & $<0.01$ \\
\hline BMI (continuous) & 0.95 & $0.93-0.97$ & $<0.01$ & 0.95 & $0.94-0.96$ & $<0.01$ \\
\hline Current smoke & 1.04 & $0.75-1.43$ & 0.83 & 1.08 & $0.93-1.24$ & 0.31 \\
\hline Diabetes mellitus & 1.74 & $1.32-2.30$ & $<0.01$ & 1.61 & $1.42-1.82$ & $<0.01$ \\
\hline Stroke & 3.28 & $2.34-4.60$ & $<0.01$ & 2.97 & $2.56-3.44$ & $<0.01$ \\
\hline Emphysema & 3.44 & $2.13-5.57$ & $<0.01$ & 3.64 & $3.00-4.41$ & $<0.01$ \\
\hline Liver disease & 0.72 & $0.36-1.46$ & 0.34 & 1.21 & $0.94-1.54$ & 0.14 \\
\hline Malignant tumor & 1.95 & $1.43-2.65$ & $<0.01$ & 2.07 & $1.81-2.36$ & $<0.01$ \\
\hline Congestive heart failure & 7.12 & $5.32-9.52$ & $<0.01$ & 3.69 & $3.18-4.29$ & $<0.01$ \\
\hline Coronary heart disease & 4.49 & $3.34-6.04$ & $<0.01$ & 2.48 & $2.13-2.89$ & $<0.01$ \\
\hline Albuminuria & 3.45 & $2.65-4.49$ & $<0.01$ & 3.16 & $2.81-3.54$ & $<0.01$ \\
\hline Hemoglobin & 0.83 & $0.77-0.90$ & $<0.01$ & 0.81 & $0.78-0.84$ & $<0.01$ \\
\hline eGFR $<60 \mathrm{ml} / \mathrm{min} / 1.73 \mathrm{~m}^{2}$ & 4.28 & $3.33-5.52$ & $<0.01$ & 3.55 & $3.18-3.97$ & $<0.01$ \\
\hline CONUT (continuous) & 1.54 & $1.41-1.68$ & $<0.01$ & 1.51 & $1.46-1.58$ & $<0.01$ \\
\hline NRI (continuous) & 0.96 & $0.95-0.96$ & $<0.01$ & 0.96 & $0.95-0.96$ & $<0.01$ \\
\hline NPS (continuous) & 1.72 & $1.52-1.94$ & $<0.01$ & 1.71 & $1.62-1.81$ & $<0.01$ \\
\hline
\end{tabular}

BMI, Body Mass Index; eGFR, estimated Glomerular Filtration Rate; CONUT, Controlling Nutritional Status score; NRI, Nutritional Risk Index; NPS, Naples Prognostic Score.

TABLE 5 | Univariate and multivariate Cox regression of three nutritional screening tools for cardiovascular death and all-cause death.

\begin{tabular}{|c|c|c|c|c|c|c|c|c|}
\hline \multirow[t]{2}{*}{ Variables } & \multicolumn{4}{|c|}{ Cardiovascular death } & \multicolumn{4}{|c|}{ All-cause death } \\
\hline & HR $(95 \% \mathrm{Cl})$ & $P$ & HR (95\%Cl) & $P$ & HR (95\%Cl) & $P$ & HR (95\%Cl) & $P$ \\
\hline CONUT Normal & / & & / & & / & & / & \\
\hline Mild & $2.52(1.90-3.34)$ & $<0.01$ & $1.41(1.04-1.91)$ & 0.03 & $2.51(2.22-2.84)$ & $<0.01$ & $1.48(1.30-1.70)$ & $<0.01$ \\
\hline Mild & $1.94(1.23-3.07)$ & $<0.01$ & $1.08(0.67-1.73)$ & 0.76 & $2.40(1.91-3.02)$ & $<0.01$ & $1.65(1.30-2.08)$ & $<0.01$ \\
\hline Moderate to severe & $4.69(2.86-7.70)$ & $<0.01$ & $1.54(0.91-2.63)$ & 0.11 & $6.31(4.96-8.01)$ & $<0.01$ & $2.90(2.24-3.74)$ & $<0.01$ \\
\hline NRI Normal & / & & / & & / & & / & \\
\hline Mild & $3.89(2.17-6.96)$ & $<0.01$ & $2.60(1.34-5.07)$ & $<0.01$ & $2.64(1.96-3.54)$ & $<0.01$ & $1.72(1.24-2.39)$ & $<0.01$ \\
\hline Moderate to severe & $3.88(2.17-6.94)$ & $<0.01$ & $3.30(1.66-6.56)$ & $<0.01$ & $4.28(3.42-5.35)$ & $<0.01$ & $2.60(1.96-3.44)$ & $<0.01$ \\
\hline
\end{tabular}

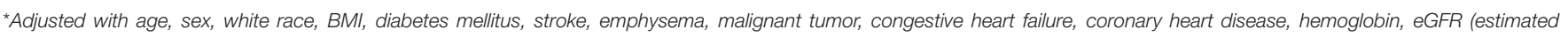
Glomerular Filtration Rate), abuminuria.

might because of different parameters included or different thresholds for the same parameter. The poor concordance in identifying malnutrition among the three screening tools suggest that they are not interchangeable. The nutritional status of hypertensive patients evaluated by CONUT and NPS suggest that malnutrition was quite common among these patients. On the one hand, it might be owing to the physiological interrelationship between hypertension and inflammation $(4,19)$. Previous studies have reported that hypertensive patients had higher plasma concentrations of proinflammatory cytokines and acute phase proteins (20).
The activation of inflammatory pathways might increase the catabolic demands and result in malnutrition. On the other hand, vitamin D deficiency was prevalent among malnourished patients (21) and its deficiency was highly associated with incidence of metabolic syndrome (22) and cardiovascular disease including hypertension (23), while vitamin D could act on endothelial cells and smooth muscle cells to regulate blood pressure (24).

The relationship between nutritional status and prognosis has been confirmed in some cardiovascular diseases. In a study including 5,062 acute coronary syndrome patients with 

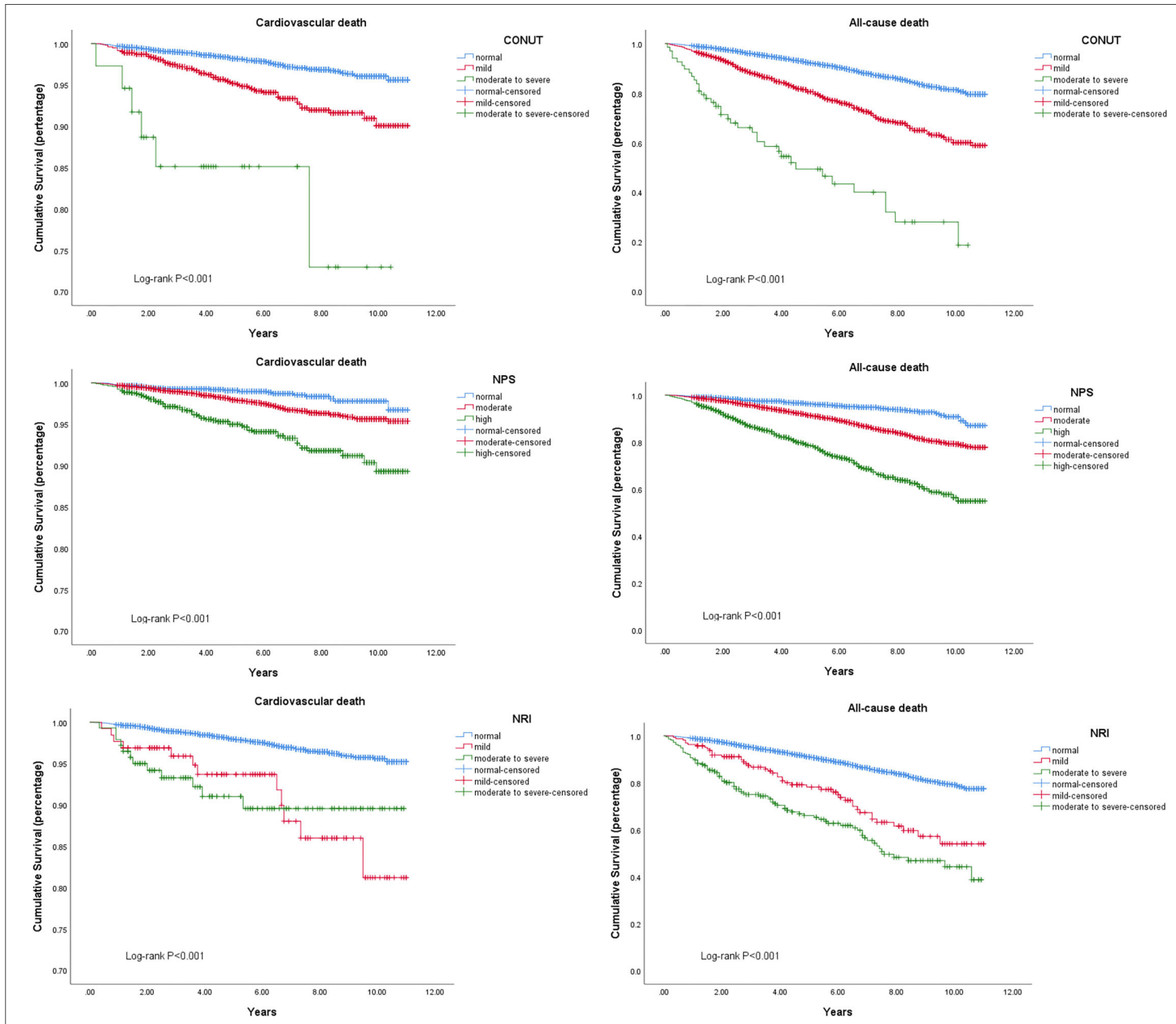

FIGURE 3 | Kaplan-Meier curves of long-term cardiovascular and all-cause mortality for different nutritional status assessed by three nutritional screening tools.

a median age of 66.2 years, multivariate cox proportional hazard regression analysis indicated that malnutrition assessed by CONUT and NRI was an independent factor for allcause mortality and cardiovascular events (25). Another study involving 336 elderly hypertensive patients confirmed that poor nutritional status assessed by the CONUT score was significantly associated with all-cause mortality (18). In the present study, malnutrition evaluated by different NSTs was also significantly associated with both cardiovascular and all-cause mortality in hypertensive patients. The possible underlying mechanism might be explained by the following facts. First, tumor necrosis factoralpha (TNF- $\alpha$ ), a key inflammatory mediator (26), was found to be higher in patients with moderate-to-severe malnutrition. The underlying inflammation process could involve the pathogenesis and progress of some cardiovascular diseases $(27,28)$ such as coronary artery disease $(29,30)$ and heart failure (31), thereby supplementing the cardiovascular risk brought on by hypertension (32) and finally leading to cardiovascular events. Second, as mentioned previously, malnutrition patient always comorbidity with vitamin D deficiency (21, 33). The deficiency of vitamin $\mathrm{D}$ was reported associated with higher risk of uncontrolled BP in hypertensive patients (34) which was associated premature vascular death and CVD mortality (35). Third, all three NSTs include serum albumin as a parameter, while hypoalbuminemia was confirmed to be associated with extremely poor prognosis and cardiac cachexia $(36,37)$.

Given that malnutrition assessed by NSTs is now a common occurrence among hypertensive patients and proved to be associated with higher cardiovascular and all-cause 


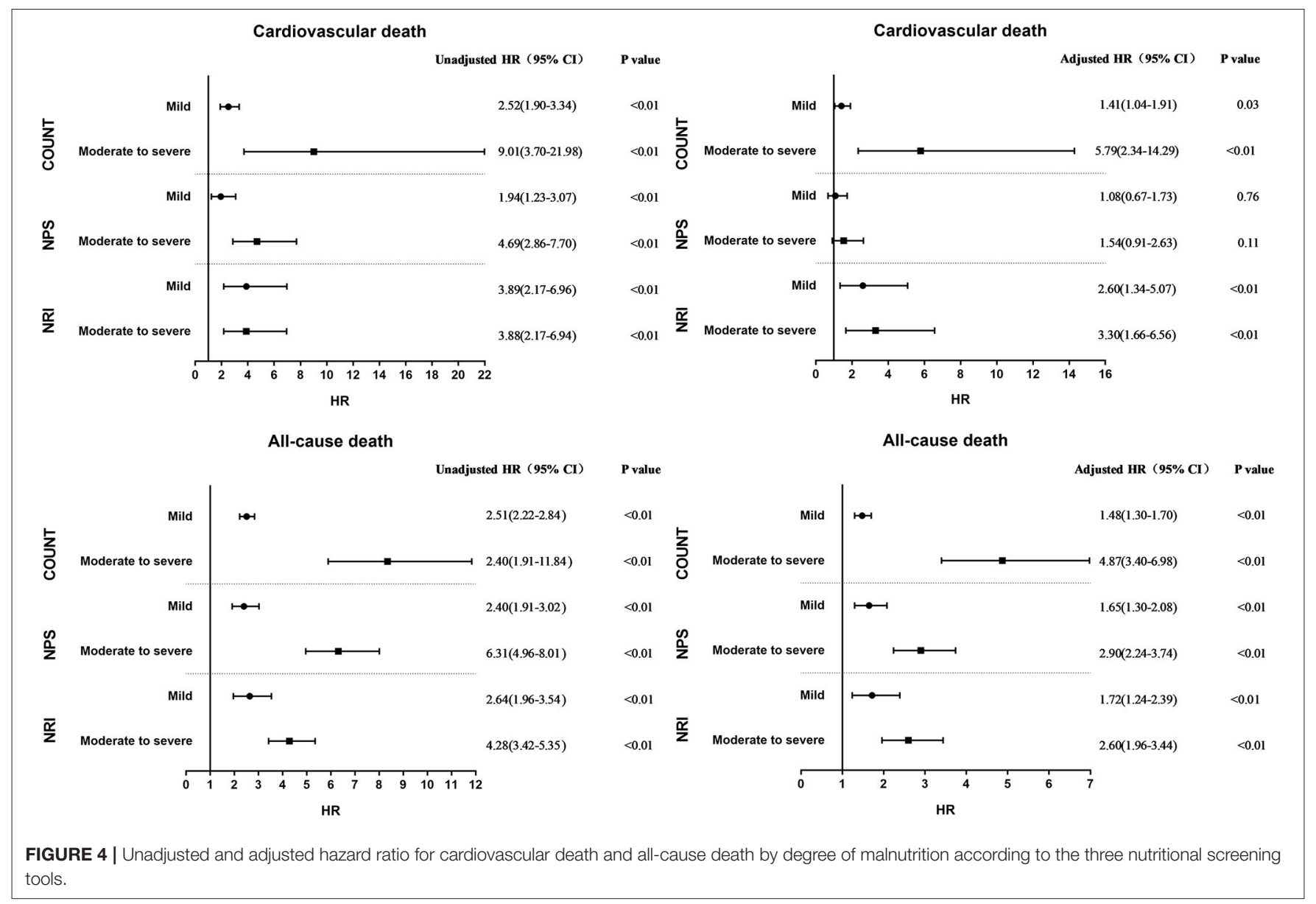

mortality, the question remains, which NST is suitable for clinicians to identify hypertensive patients with malnutrition. Our study showed that NPS was not suitable for predicting long-term cardiovascular mortality, while nutritional status evaluated by NPS was significantly associated with all-cause mortality but not cardiovascular mortality after adjustment for potential confounding factors. This phenomenon might be due to the threshold setting of its parameters. In the NPS scoring system (14), patients with serum albumin $<40 \mathrm{~g} / \mathrm{L}$ were considered to have malnutrition, while the threshold for serum albumin was $35 \mathrm{~g} / \mathrm{L}$ in the CONUT scoring system (12), which led to its poor ability to distinguish patients with and without malnutrition. Although nutritional status assessed by NRI was significantly associated with both all-cause mortality and cardiovascular mortality, it showed poorer performance than CONUT in differentiating patients with mild and moderate-to-severe malnutrition in terms of mortality risk; this could be explained by the small number of patients with malnutrition evaluated by NRI. Moreover, NRI was recommended for identify malnutrition among elderly hypertensive patients or with comorbidities, while the NRI had advantages on predicting both cardiovascular mortality and all-cause mortality among elderly hypertensive patients or with comorbidities. Overall, CONUT was a more suitable
NST than NPS and NRI to identify malnutrition among all hypertensive patients.

\section{LIMITATION}

There were some limitations to this study that should be noted. First, the proportion of patients who were classified with moderate-to-severe malnutrition was low leading to the limited value of this study for those hypertensive patients with extremely poor nutritional status. Second, because of the retrospective study design, our findings should be interpreted with caution. Finally, although the models were adjusted for potential risk factors using multiple regression analysis techniques, there may have been some residual confounding factors.

\section{CONCLUSION}

Malnutrition evaluated by NSTs was common among hypertensive patients and was closely associated with both longterm cardiovascular mortality and all-cause mortality. Clinicians should make additional efforts for the early identification and management of malnutrition. 


\section{DATA AVAILABILITY STATEMENT}

The original contributions presented in the study are included in the article/Supplementary Material, further inquiries can be directed to the corresponding author/s.

\section{ETHICS STATEMENT}

The studies involving human participants were reviewed and approved by Ethics Review Committee of NCHS of the Centers for Disease Control and Prevention. The patients/participants provided their written informed consent to participate in this study. Written informed consent was obtained from the individual(s), and minor(s)' legal guardian/next of kin, for the publication of any potentially identifiable images or data included in this article.

\section{AUTHOR CONTRIBUTIONS}

Z-wY, X-bW, D-qY, and J-yC contributed to the conception or design of the study. Z-wY contributed to the acquisition, analyses,

\section{REFERENCES}

1. Freeman AM, Morris PB, Barnard N, Esselstyn CB, Ros E, Agatston A, et al. Trending cardiovascular nutrition controversies. J Am Coll Cardiol. (2017) 69:1172-87. doi: 10.1016/j.jacc.2016.10.086

2. Saxton SN, Clark BJ, Withers SB, Eringa EC, Heagerty AM. Mechanistic links between obesity, diabetes, and blood pressure: role of perivascular adipose tissue. Physiol Rev. (2019) 99:1701-63. doi: 10.1152/physrev.00034. 2018

3. Goldfarb M, Lauck S, Webb JG, Asgar AW, Perrault LP, Piazza $\mathrm{N}$, et al. Malnutrition and mortality in frail and non-frail older adults undergoing aortic valve replacement. Circulation. (2018) 138:2202-11. doi: 10.1161/circulationaha.118.033887

4. Sze S, Pellicori P, Kazmi S, Rigby A, Cleland JGF, Wong K, et al. Prevalence and prognostic significance of malnutrition using 3 scoring systems among outpatients with heart failure: a comparison with body mass index. JACC Heart Fail. (2018) 6:476-86. doi: 10.1016/j.jchf.2018.02.018

5. Raposeiras-Roubín S, Abu-Assi E, Paz RC, Rosselló X, Barreiro Pardal C, Piñón Esteban M., et al. Impact of malnutrition in the embolic-haemorrhagic trade-off of elderly patients with atrial fibrillation. Europace. (2020) 22:87887. doi: 10.1093/europace/euaa017

6. Mills KT, Bundy JD, Kelly TN, Reed JE, Kearney PM, Reynolds K, et al. Global disparities of hypertension prevalence and control: a systematic analysis of population-based studies from 90 countries. Circulation. (2016) 134:44150. doi: 10.1161/circulationaha.115.018912

7. Zhou D, Xi B, Zhao M, Wang L, Veeranki SP. Uncontrolled hypertension increases risk of all-cause and cardiovascular disease mortality in US adults: the NHANES III linked mortality study. Sci Rep. (2018) 8:9418. doi: 10.1038/s41598-018-27377-2

8. Zhou B, Perel P, Mensah GA, Ezzati M. Global epidemiology, health burden and effective interventions for elevated blood pressure and hypertension. Nat Rev Cardiol. (2021) 18:785-802. doi: 10.1038/s41569-021-00559-8

9. Craig LS, Gage AJ, Thomas AM. Prevalence and predictors of hypertension in Namibia: a national-level cross-sectional study. PLoS ONE. (2018) 13:e0204344. doi: 10.1371/journal.pone.0204344

10. Muntner P, Hardy ST, Fine LJ, Jaeger BC, Wozniak G, Levitan EB, et al. Trends in blood pressure control among US adults with hypertension, 19992000 to 2017-2018. Jama. (2020) 324:1190-200. doi: 10.1001/jama.2020. 14545 and interpretation of data. Z-wY, X-bW, and B-qF drafted the manuscript. D-qY revised the manuscript critically, had all access to the data, and is responsible for the overall content as guarantor. All authors contributed to refinement of the study protocol and approved the final manuscript.

\section{FUNDING}

This work was supported by grants from National Natural Science Foundation of China (Grant No. 82002014), Natural Science Foundation of Guangdong Province (Grant No. 2021A1515010107), and Science and Technology Projects of Guangzhou (Grant No. 201903010097). The funders had no role in the study design, data collection and analysis, decision to publish, nor preparation of the manuscript.

\section{SUPPLEMENTARY MATERIAL}

The Supplementary Material for this article can be found online at: https://www.frontiersin.org/articles/10.3389/fnut.2022. 822376/full\#supplementary-material

11. Centers for Disease Control and Prevention, NCHS. National Health and Nutrition Examination Survey Data. (2020). Available online at: https://www. cdc.gov/nchs/nhanes/ (accessed September 1, 2020).

12. Wada H, Dohi T, Miyauchi K, Doi S, Konishi H, Naito R, et al. Prognostic impact of nutritional status assessed by the Controlling Nutritional Status score in patients with stable coronary artery disease undergoing percutaneous coronary intervention. Clin Res Cardiol. (2017) 106:87583. doi: 10.1007/s00392-017-1132-z

13. Bouillanne O, Morineau G, Dupont C, Coulombel I, Vincent JP, Nicolis I, et al. Geriatric nutritional risk index: a new index for evaluating at-risk elderly medical patients. Am J Clin Nutr. (2005) 82:777-83. doi: 10.1093/ajcn/82.4.777

14. Galizia G, Lieto E, Auricchio A, Cardella F, Mabilia A, Podzemny V, et al. Naples prognostic score, based on nutritional and inflammatory status, is an independent predictor of long-term outcome in patients undergoing surgery for colorectal cancer. Dis Colon Rectum. (2017) 60:127384. doi: $10.1097 /$ dcr.0000000000000961

15. Micallef L, Rodgers P. eulerAPE: drawing area-proportional 3-Venn diagrams using ellipses. PLoS ONE. (2014) 9:e101717. doi: 10.1371/journal.pone.0101717

16. Savica V, Bellinghieri G, Kopple JD. The effect of nutrition on blood pressure. Annu Rev Nutr. (2010) 30:365401. doi: 10.1146/annurev-nutr-010510-103954

17. Lennon SL, DellaValle DM, Rodder SG, Prest M, Sinley RC, Hoy MK, et al. 2015 evidence analysis library evidence-based nutrition practice guideline for the management of hypertension in adults. J Acad Nutr Diet. (2017) 117:1445.e17-58.e17. doi: 10.1016/j.jand.2017.04.008

18. Sun X, Luo L, Zhao X, Ye P. Controlling Nutritional Status (CONUT) score as a predictor of all-cause mortality in elderly hypertensive patients: a prospective follow-up study. BMJ Open. (2017) 7:e015649. doi: 10.1136/bmjopen-2016-015649

19. Nakagomi A, Kohashi K, Morisawa T, Kosugi M, Endoh I, Kusama Y, et al. Nutritional status is associated with inflammation and predicts a poor outcome in patients with chronic heart failure. J Atheroscler Thromb. (2016) 23:713-27. doi: 10.5551/jat.31526

20. Guzik TJ, Touyz RM. Oxidative stress, inflammation, and vascular aging in hypertension. Hypertension. (2017) 70:6607. doi: 10.1161/hypertensionaha.117.07802

21. Merker M, Amsler A, Pereira R, Bolliger R, Tribolet P, Braun N, et al. Vitamin $\mathrm{D}$ deficiency is highly prevalent in malnourished inpatients and associated 
with higher mortality: a prospective cohort study. Medicine (Baltimore). (2019) 98:e18113. doi: 10.1097/md.0000000000018113

22. Wieder-Huszla S, Jurczak A, Szkup M, Barczak K, Dołegowska B, SchneiderMatyka D, et al. Relationships between vitamin D3 and metabolic syndrome. Int J Environ Res Public Health. (2019) 16:175. doi: 10.3390/ijerph16020175

23. Latic N, Erben RG. Vitamin D and cardiovascular disease, with emphasis on hypertension, atherosclerosis, and heart failure. Int J Mol Sci. (2020) 21:6483. doi: 10.3390/ijms21186483

24. de la Guía-Galipienso F, Martínez-Ferran M, Vallecillo N, Lavie CJ, SanchisGomar F, Pareja-Galeano H. Vitamin D and cardiovascular health. Clin Nutr. (2021) 40:2946-57. doi: 10.1016/j.clnu.2020.12.025

25. Raposeiras Roubín S, Abu Assi E, Cespón Fernandez M, Barreiro Pardal C, Lizancos Castro A, Parada JA, et al. Prevalence and prognostic significance of malnutrition in patients with acute coronary syndrome. J Am Coll Cardiol. (2020) 76:828-40. doi: 10.1016/j.jacc.2020.06.058

26. Tonet E, Campo G, Maietti E, Formiga F, Martinez-Sellés M, Pavasini R, et al. Nutritional status and all-cause mortality in older adults with acute coronary syndrome. Clin Nutr. (2020) 39:1572-9. doi: 10.1016/j.clnu.2019. 06.025

27. Golia E, Limongelli G, Natale F, Fimiani F, Maddaloni V, Pariggiano I, et al. Inflammation and cardiovascular disease: from pathogenesis to therapeutic target. Curr Atheroscler Rep. (2014) 16:435. doi: 10.1007/s11883-0140435- $\mathrm{z}$

28. Liu HH, Cao YX, Sun D, Jin JL, Zhang HW, Guo YL, et al. Highsensitivity $\mathrm{C}$-reactive protein and hypertension: combined effects on coronary severity and cardiovascular outcomes. Hypertens Res. (2019) 42:178393. doi: 10.1038/s41440-019-0293-8

29. Ali L, Schnitzler JG, Kroon J. Metabolism: The road to inflammation and atherosclerosis. Curr Opin Lipidol. (2018) 29:474-80. doi: 10.1097/mol.0000000000000550

30. Wang $\mathrm{H}$, Liu Z, Shao J, Lin L, Jiang M, Wang L, et al. Immune and inflammation in acute coronary syndrome: molecular mechanisms and therapeutic implications. J Immunol Res. (2020) 2020:4904217. doi: 10.1155/2020/4904217

31. Adamo L, Rocha-Resende C, Prabhu SD, Mann DL. Reappraising the role of inflammation in heart failure. Nat Rev Cardiol. (2020) 17:26985. doi: 10.1038/s41569-019-0315-x
32. Kjeldsen SE. Hypertension and cardiovascular risk: general aspects. Pharmacol Res. (2018) 129:95-9. doi: 10.1016/j.phrs.2017.11.003

33. Agarwal A, Gupta SK, Sukumar R. Hyperparathyroidism and malnutrition with severe vitamin D deficiency. World J Surg. (2009) 33:2303-13. doi: 10.1007/s00268-009-0044-0

34. Del Pinto R, Wright JT, Monaco A, Pietropaoli D, Ferri C. Vitamin D and blood pressure control among hypertensive adults: results from NHANES 2001-2014. J Hypertens. (2020) 38:150-8. doi: 10.1097/hjh.0000000000002231

35. Dan H, Kim J, Kim O. Effects of gender and age on dietary intake and body mass index in hypertensive patients: analysis of the Korea national health and nutrition examination. Int J Environ Res Public Health. (2020) 17:4482. doi: 10.3390/ijerph17124482

36. Rahman A, Jafry S, Jeejeebhoy K, Nagpal AD, Pisani B, Agarwala R. Malnutrition and cachexia in heart failure. JPEN J Parenter Enteral Nutr. (2016) 40:475-86. doi: 10.1177/0148607114566854

37. Corsetti, G., Pasini, E., Romano, C., Chen-Scarabelli, C., Scarabelli, T. M., Flati, V., et al. (2021). How can malnutrition affect autophagy in chronic heart failure? Focus and perspectives. Int $J$ Mol Sci 22:3332. doi: $10.3390 /$ ijms 22073332

Conflict of Interest: The authors declare that the research was conducted in the absence of any commercial or financial relationships that could be construed as a potential conflict of interest.

Publisher's Note: All claims expressed in this article are solely those of the authors and do not necessarily represent those of their affiliated organizations, or those of the publisher, the editors and the reviewers. Any product that may be evaluated in this article, or claim that may be made by its manufacturer, is not guaranteed or endorsed by the publisher.

Copyright $\odot 2022$ Yang, Wei, Fu, Chen and Yu. This is an open-access article distributed under the terms of the Creative Commons Attribution License (CC BY). The use, distribution or reproduction in other forums is permitted, provided the original author(s) and the copyright owner(s) are credited and that the original publication in this journal is cited, in accordance with accepted academic practice. No use, distribution or reproduction is permitted which does not comply with these terms. 